\title{
Estados inmaduros de Leptoglossus zonatus (Hemiptera, Coreidae): agente relacionado con la caída de naranjas (Citrus sinensis) en Azuero, Panamá
}

Immature stages of Leptoglossus zonatus (Hemiptera, Coreidae): agent related with the fall of orange fruits (Citrus sinensis) in Azuero, Panama

\author{
Rubén Collantes González ${ }^{1}$, Pedro Rodríguez ${ }^{1}$, Bolívar Romero ${ }^{1}$ Y \\ ERICK Rodríguez ${ }^{1}$
}

\section{RESUMEN}

Se presenta una descripción detallada de huevos y ninfas de Leptoglossus zonatus Dallas, 1852. Se obtuvo especímenes colectados de traspatios con naranjos en las provincias de Herrera y Los Santos, las cuales conforman la región de Azuero, Panamá. Mediante claves taxonómicas de identificar de 32 especímenes adultos de $L$. zonatus, y se seleccionaron cuatro para ser ubicados en cámaras de cría con naranjas lavadas como fuente de alimentación y sustrato de oviposición. Se observó y tomó fotografías para describir cabeza, tórax y abdomen de las formas inmaduras, las cuales sobrevivieron hasta el tercer estadio. Los huevos son de color marrón, dorsalmente cubiformes, ordenados en hileras continuas de más de 10 unidades. Las ninfas son amarillo brillante, anaranjado y negro, con ojos rojos prominentes, setas dispersas y proyecciones dorso-laterales equidistantes.

Palabras clave: coreidae; huevo; naranja; ninfa; Panamá.

\begin{abstract}
A detailed description of eggs and nymphs of Leptoglossus zonatus Dallas, 1852 is presented. Previously, there were specimens collected from backyard with orange in the provinces of Herrera and Los Santos, which conform the Azuero Region, Panama. Using taxonomic keys of Brailovsky \& Barrera (1998), to confirm the identity of 32 adult specimens of $L$. qonatus, from which four specimens were placed in rearing chambers with washed oranges as food and oviposition substrate. Observation, photograph taking and description immature forms head, thorax and abdomen was made and it was determine that they only survive until the third stage. Eggs are brown, cubic dor-
\end{abstract}

1 Universidad de «Panamá». Panamá, Panamá. 
sally, arranged in continuous rows of more than 10 units. Nymphs are bright yellow, orange and black, with bulging red eyes, scattered hairs and dorsolateral equidistant projections.

Keywords: coreidae; egg; orange; nymph; Panama.

\section{ICHIKLLACHAW}

Kaychawqa rurukunawan ninfakuna Leptoglossus zonatus Dallaspa nishqantam alliq riqitsikun, 1852. Tsaypaq paqtakashqa ispisiminis nishqantam hurqakashqa, naranhakunawan transpatiyuspiq Herrerawan Los Santos suyukunachaw, kaykuna Azuero suyu, Panamachawmi kayan. Kayqa Brailovskywan Barrera (1998) kanqanraykurmi rurakashqa, kaychaw 32 puqushqa ispisiminis $L$ zonatus nishqantam riqikashqa, hinaman chuskutam akrakashqa wataypa kamarasninchaw paqashqa narahakunawan mikuynin alli kaptin, hina oviposiciónpa sustratunchaw. Qawaqashqa niykur hutukashqa piqanta, qasqu tulluwan pachan kaqninta kanqanta ninapaq, kaykuna kima kaq istadiyukama kawayashqanta. Rurukunaqa paqu llimpim kayan, waqtan kaqqa kubihurmikuna rikuq kayan, qatinakushqalla ñiqinqashqa chunkapitapis atskayashqa. Ninhuwakunaqa chipapiq qarwash, ayawmawan yanam kayan, puka ñawikunawan, setaskuna rakikashqawan waqtan kaq kuchunqa qaqantsashqam kayan.

Pushaq shimikuna: kurayday; ruru; naranha; ninhuwa; Panama.

\section{INTRODUCCIÓN}

El género Leptoglossus Guérin-Méneville, 1831 pertenece a la familia Coreidae (Hemiptera, Heteroptera), llamados comúnmente chinches patas de hoja. Son de amplia distribución en el Neotrópico y se conocen, según Brailovsky (2014), 61 especies y una subespecie descritas, de las cuales L. qonatus es, de acuerdo con Brailovsky y Barrera (2004), una especie altamente polífaga que ocasiona problemas en cultivos como maíz, sorgo, algodón, guayaba, palto, pasifloras, plátano, berenjena, tomate, cucurbitáceas, leguminosas, sésamo, durazno, pecana, granada, piñón blanco, pitahaya amarilla, marañón, carambola, cítricos, entre muchos otros.

La importancia de esta plaga se ve reflejada en los trabajos desarrollados por Schaefer y Mitchell (1983), Panizzi (1989; 2004), Kubo y Batista (1992), Maes y Goellner-Scheiding (1993), Matrangolo y Waquil (1994), Grimm y Maes (1997), Souza y Amaral (1999a; b), Brailovsky (2014), Brailovsky y Barrera (1998; 2004), Díaz y Orellana (2000), Mitchell (2000), OIRSA (2000), Campos y Echeverría (2004), Duarte (2006), Tarango et al. (2007), Duarte et al. (2008), Jiménez-Martínez y Gómez (2011), Pires et al. (2011), Rodríguez et al. (2011), Chi y Mizell (2012), Yepes et al. (2012), Kondo et al. (2013), López et al. (2013), Tarango (2013), Alonso y Lezcano (2014).

En dichas publicaciones, se aborda aspectos como la biología, distribución geográfica, rango de hospedantes, enemigos naturales, métodos de control y taxonomía, siendo la última por lo general desarrollada con insectos adultos. Sin embargo, reconocer estados inmaduros facilita el desarrollo de alternativas de control más oportunas frente a problemáticas a las cuales se asocia L. zonatus. Por lo expuesto, el presente trabajo tiene por objetivo describir huevos y ninfas de L. zonatus, agente relacionado con la caída de frutos de naranja en la Región de Azuero, Panamá. 


\section{MATERIALES Y MÉTODOS}

Se recolectaron 32 especímenes adultos de L. zonatus asociados a cultivos de naranja, frijol y ají en las provincias de Herrera y Los Santos, Península de Azuero, Panamá (figura 1).

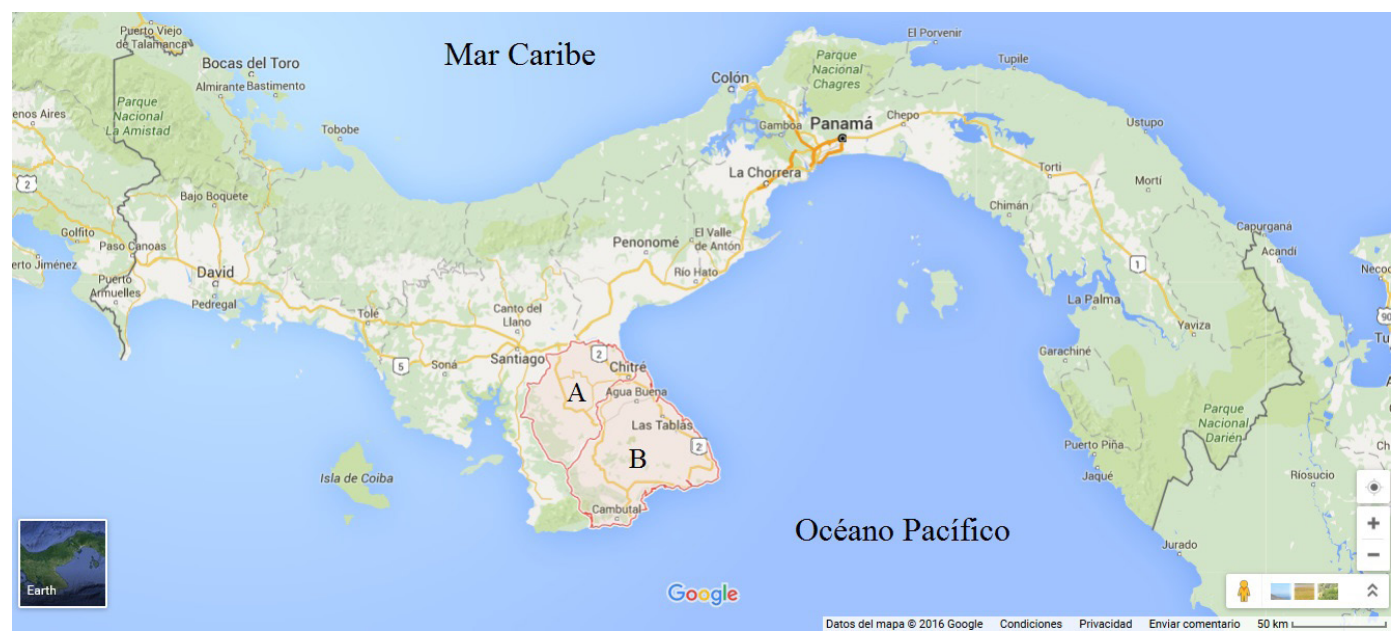

Figura 1. Península de Azuero, Panamá: A) Provincia de Herrera; B) Provincia de Los Santos.

Fuente: https://www.google.com.pe/maps

La fase de laboratorio se desarrolló en el Programa Centroamericano de Postgrado en Entomología, Universidad de Panamá. Se consultó los trabajos de Brailovsky y Barrera (1998) y Brailovsky (2014), para confirmar la identidad de la especie mediante caracteres morfológicos externos e internos (figura 2). Posteriormente, se seleccionó dos parejas de adultos y se colocaron en cámaras de cría, constituidas por cilindros traslúcidos de $12 \mathrm{~cm}$ de diámetro por $20 \mathrm{~cm}$ de alto, tapadas con tul.

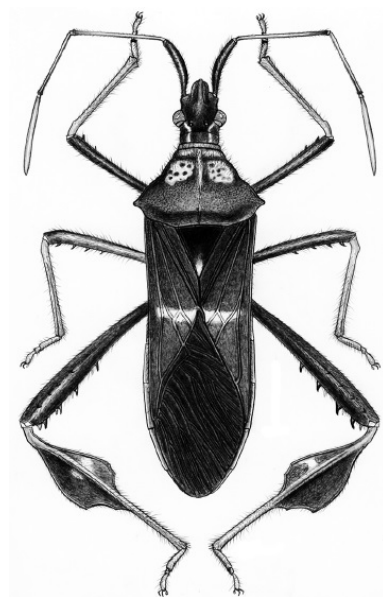

Figura 2. L. qonatus ठึ

Fuente: Brailovsky (2014)

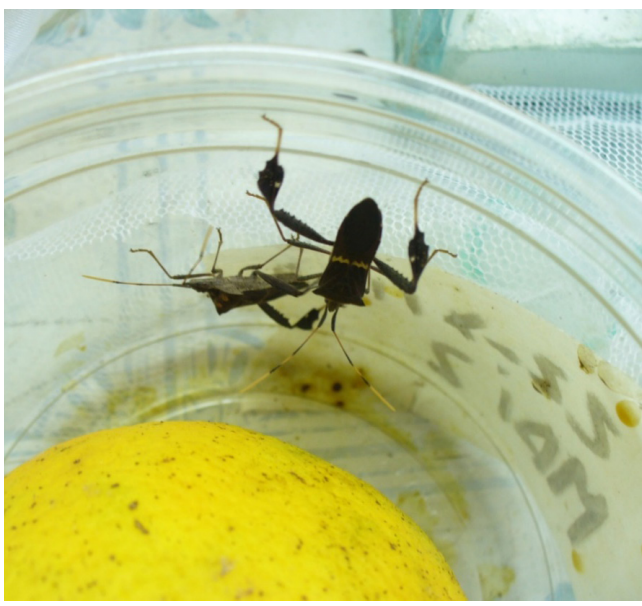

Figura 3. Cámara de cría de L. zonatus. 
La temperatura fue de $28 \pm 2^{\circ} \mathrm{C}$, con fotoperíodo regular de $12 \mathrm{~h}$ luz: $12 \mathrm{~h}$ oscuridad. Como sustrato de alimentación y oviposición, se colocó un fruto de naranja, lavado y seco, por cámara (Figura 3). Se observó durante un mes, período en el cual las hembras colocaron posturas y emergieron ninfas.

\section{RESULTADOS}

De acuerdo con las observaciones realizadas en laboratorio, se procede a detallar los estados inmaduros de Leptoglossus ronatus:

- Huevos (figura 3): ordenados en hileras de hasta 20 unidades, inicialmente de color amarillo oscuro, tornándose amarillo ocre conforme maduran. De aspecto cubiforme en vista dorsal, a modo de barril comprimido. Se encuentran unidos anterior y posteriormente al siguiente mediante una resina marrón. El operculum (tapita), es ovoide y se ubica en el extremo anterior de cada huevo, con proceso micropilar liso.
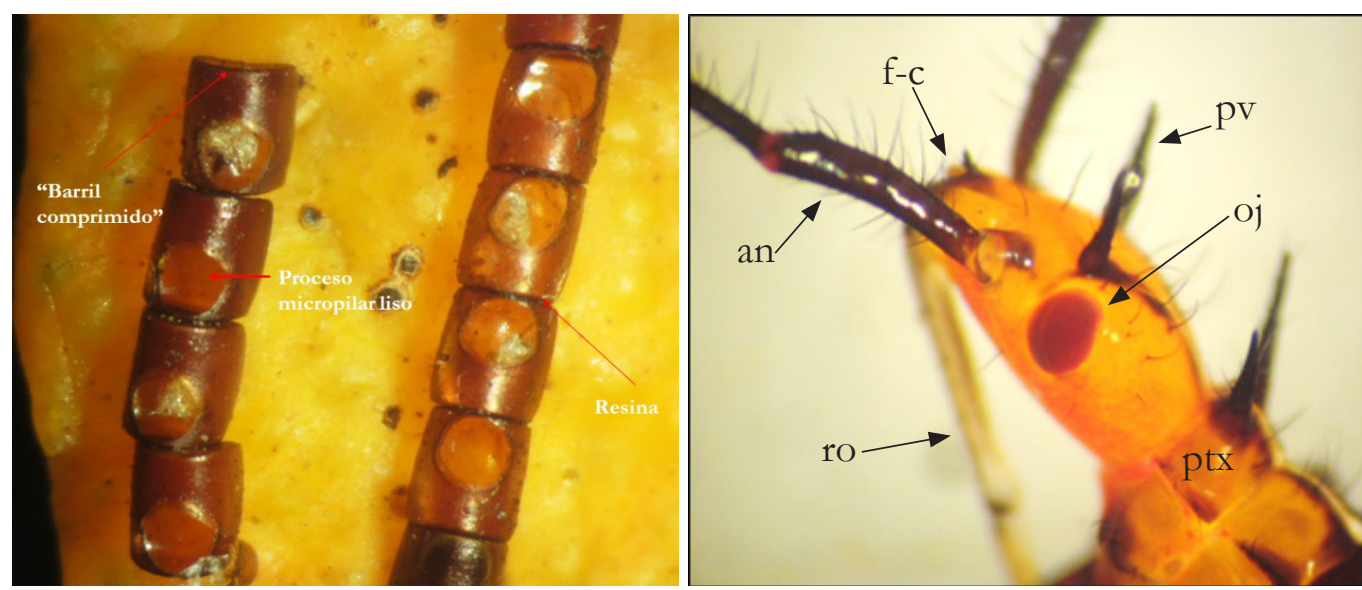

Figura 3. Huevos de L. zonatus Figura 4. Cabeza de ninfa, L. zonatus

- Ninfas: entre 5-13 mm durante los estados ninfales 1-3. Cabeza (figura 4), amarilla, brillante, lustrosa con setas dispersas; ojos (oj), rojos prominentes; juga y tylus con longitud similar, rostrum (ro), extendiéndose hasta el último segmento abdominal; dos proyecciones negras en el vertex (pv); antenas de cuatro segmentos (an), pilosas; fronto-clypeus (f-c), con tres setas formando un triángulo.

Tórax (figura 5), amarillo con bordes laterales naranja oscuro, setas dispersas; patas negras, tan largas como la longitud del cuerpo, con parches naranja dispersos; tarsi bi-segmentado; tecas alares (en ninfa 3); dos proyecciones dorsales negras en el protórax (ptx); protórax, meso (mst) y metatórax (mtt), con espiráculos (sp), situados en el margen posterior.

Abdomen (figura 6), amarillo y naranja, con aberturas odoríferas situadas entre los segmentos 4-5 y 5-6, con proyecciones dorso-laterales negras (pdl), equidistantes a cada abertura; espiráculos circulares (sp), desde el segundo segmento hasta el octavo segmento, situados latero-ventralmente; últimos terga abdominales fuertemente esclerosados (uta). 

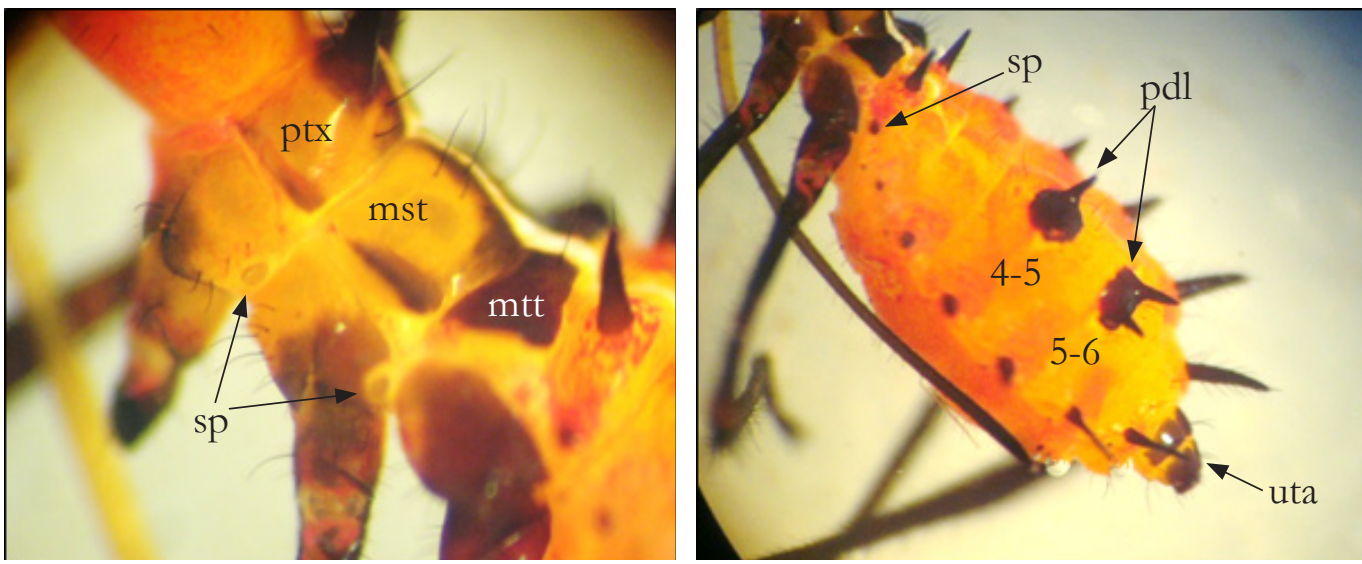

Figura 5. Tórax, ninfa de L. zonatus / Figura 6. Abdomen, ninfa de L. zonatus

\section{DISCUSIÓN}

Las características externas observadas en los huevos se asemejan a lo descrito para otras especies cercanas, de la tribu Anisoscelini, como Narnia femorata Stal y Phthia picta (Drury), detalladas en trabajos de Brailovsky et al. (1994) y Silva et al. (2001), respectivamente. Por tanto, el estado de huevo podría servir para identificar tribus, pero no géneros ni mucho menos especies. Adicionalmente, Matrangolo y Waquil (1994), afirmaron que en promedio cada hembra puede poner entre cinco y seis posturas con 15 huevos distribuidos en una sola línea, lo cual coincide con lo observado en las hembras estudiadas, las cuales colocaron posturas con 10 a 20 unidades.

La no sobrevivencia del tercer estado ninfal confirma los resultados obtenidos por Duarte et al. (2008), en los cuales el 100\% de la mortalidad de las ninfas de L. zonatus se alcanzó al final del segundo estado ninfal. Ello justifica el empleo de dietas suplementarias para su crianza en condiciones de laboratorio, tal como sugieren las experiencias de Panizzi (1989), Matrangolo y Waquil (1994) y Duarte et al. (2008), en las cuales se empleó maíz, sorgo y frijol hasta que lograsen el tercer estado ninfal. Adicionalmente, ello explica la alternancia de plantas hospedantes en campo, lo cual se registró en los datos de colecta de especímenes adultos por Rodríguez et al. (2011).

En los estados ninfales 1 y 2 no se observó presencia de la expansión típica de las metatibias, lo cual coincide con lo descrito por Chi y Russell (2012). Por otro lado, de realizarse futuros estudios comparativos de estados inmaduros con otras especies de la tribu Anisoscelini, como los desarrollados por Rodrigues y Moreira (2005), difícilmente podrían apreciarse diferencias significativas en los dos primeros estados ninfales, lo cual motiva a mejorar las metodologías de crianza en laboratorio. Finalmente, varias de las características observadas en los estados inmaduros de L. zonatus son similares a los descritos por Brailovsky et al. (1994), para N. femorata (Anisoscelini).

\section{CONCLUSIONES}

Se describen los huevos y ninfas de L. zonatus, de los cuales los primeros son marrones, dorsalmente cubiformes y ordenados en hileras continuas de más de 10 unidades; y las 
segundas son de color amarillo brillante, anaranjado y negro, con ojos rojos prominentes, setas dispersas y proyecciones dorso-laterales equidistantes.

La no sobrevivencia del estado ninfal 3 confirma la necesidad de L. zonatus por hospedantes alternos para completar su desarrollo. Por lo expuesto, se concluye que los caracteres morfológicos externos observados en estados inmaduros L. zonatus permiten una aproximación hacia tribu y género, pero difícilmente hacia especie.

\section{AGRADECIMIENTOS}

A la memoria del Dr. Cheslavo A. Korytkowski G., por su apoyo en la presente investigación. Al personal de OIRSA Panamá, por suministrar especímenes y la logística en campo.

\section{REFERENCIAS BIBLIOGRÁFICAS}

Alonso, O. y Lezcano, J. C. 2014. Artrópodos asociados a Jatropha curcas Linnaeus. Funciones y estrategia para su manejo. Pastos y forrajes 37(1): 3-16.

Brailovsky, Harry. 2014. Illustrated key for identification of the species included in the genus Leptoglossus (Hemiptera: Heteroptera: Coreidae: Coreinae: Anisoscelini), and descriptions of five new species and new synonyms. Zootaxa 3794(1): 143178.

Brailovsky, Harry y Barrera, Ernesto.1998. A review of the Costa Rican species of Leptoglossus Guérin-Méneville, with descriptions of two new species (Hemiptera: Heteroptera: Coreidae). Proceedings of the California Academy of Sciences 50(6): 167-184.

Brailovsky, Harry y Barrera, Ernesto. 2004. Six new species of Leptoglossus Guérin (Hemiptera: Heteroptera: Coreidae) and epicarp lesion on pistachio fruits. J. Econ. Entomol. 77: 1163-1165.

Brailovsky, Harry; Barrera, Ernesto; Mayorga, Cristina y Ortega, Guillermina. 1994. Estadios ninfales de los coreidos del valle de Tehuacán, Puebla. (Hemiptera-Heteroptera) I. Chelinidea staffilesi, C. tabulata y Narnia femorata. Anales Inst. Biol. Univ. Nac. Autón. México, Ser. Zool. 65(2): 241-264.

Campos, Ada y Echeverría, Cristina. 2004. Evaluación de la actividad tóxica de seis plaguicidas naturales sobre la chinche pata de hoja Leptoglossus zonatus que dañan el falso fruto del marañón Anacardium occidentale. Trabajo de graduación de Licenciatura en Química y Farmacia. Universidad de El Salvador, SV. 96.

Chi, Amelio y Mizell, Russell. 2012. Western Leaffooted Bug Leptoglossus ronatus (Dallas) (Insecta: Hemiptera: Coreidae). University of Florida. IFAS Exntesion, ENNY-517. 4.

Díaz, José y Orellana, Pedro. 2000. Evaluación de extractos botánicos para el control de la chinche (Leptoglossus ronatus) del fruto del marañón (Anacardium occidentale) en el Departamento de San Miguel, El Salvador. Tesis de Ingeniero Agrónomo. Universidad de El Salvador, SV. 95. 
Duarte, Irwin. 2006. Biología, parasitoides y daños de Leptoglossus qonatus (Dallas) y Leptoglossus gonagra (Fabricius) en cultivos de Citrus spp. Trabajo de grado, Biólogo. Universidad Industrial de Santander, Bucaramanga, CO. 33.

Duarte, Irwin; Núñez, Ligia; Peña, Jorge y Pinto, Libardo. 2008. Biology, parasitoids and damage of Leptoglossus zonatus and Leptoglossus gonagra (Heteroptera: Coreidae) on Citrus. Proc. Fla. State Hort. Soc. 121: 5-9.

Grimm, Christoph y Maes, Jean-Michel. 1997. Insectos asociados al cultivo de tempate (Jatropha curcas) en el Pacífico de Nicaragua. III. Coreoidea (Heteroptera). Rev. Nica. Ent. 42: 15-34.

Jiménez-Martínez, Edgardo y Gómez, Jorge. 2011. Insecticidas botánicos y biológicos en el manejo del chinche patas de hoja (Leptoglossus ronatus, Dallas. Hemiptera: Coreidae) y la mosquita negra (Trigona silvestrianum, Vachall, Himenoptera: Apidae) y su efecto sobre los enemigos naturales. Cultivo de Marañón (Anacardium occidentale L.), León, Nicaragua. La Calera 11(17): 14-25.

Kondo, Takumasa; Quintero, Edgar; Medina, Jorge; Imbachi, Karol; Delgado, Alexandra y Manrique, Marilyn. 2013. Insectos plaga de importancia económica en el cultivo de pitaya amarilla. En: T. Kondo, M. Martínez, J. Medina, A. Rebolledo y C. Cardozo (eds.) Manual Técnico: Tecnología para el manejo de pitaya amarilla Selenicereus megalanthus (K. Schum. ex. Vaupel) Moran en Colombia. CORPOICA, Valle del Cauca, CO. 96.

Kubo, R y Batista, F. 1992. Ocorrencia e danos provocados por Leptoglossus zonatus (Dallas, 185) (Hemiptera: Coreidae) em Citrus spp. An. Soc. Entomol. Brasil 21: 467-470.

López, Guillermo; Gómez, Jaime; Barrera, Juan; Herrera, Elizabeth; Hernández, Marianguadalupe; Bravo, Ernesto y Zamarripa, Alfredo. 2013. Artrópodos asociados a piñón (Jatropha curcas L.) en el sur de México. Folleto técnico No. 29. Instituto Nacional de Investigaciones Forestales, Agrícolas y Pecuarias (INIFAP), Chiapas, MX. 73.

Maes, Jean-Michel y Goellner-Scheiding, U. 1993. Catálogo de los Coreoidea (Heteroptera) de Nicaragua. Rev. Nica. Ent. 25: 1-19.

Matrangolo, Walter y Waquil, José. 1994. Biología de Leptoglossus zonatus (Dallas) (Hemiptera: Coreidae) alimentados com milho e sorgo. An. Soc. Entomol. Brasil. 23(3): 419-423.

Mitchell, Paula. 2000. Leaf-footed bugs (Coreidae). P. 337-403. En: C. W. Schaefer y A. R. Panizzi (eds.). Heteroptera of economic importance. CRC Press. Boca Raton, FL.

OIRSA (Organismo Internacional Regional de Sanidad Agropecuaria). 2000. Manual técnico: Buenas prácticas de cultivo en pitahaya. 54.

Panizzi, Antonio. 1989. Desempenho de ninfas e adultos de Leptoglossus zonatus Dallas (Hemiptera: Coreidae) em diferentes alimentos. An. Soc. Entomol. Brasil 18: 375-389. 
Panizzi, Antonio. 2004. A possible territorial or recognition behavior of Leptoglossus zonatus (Dallas) (Heteroptera, Coreidae). Revista Brasileira de Entomologia 48(4): 577-579.

Pires, Evaldo; Bonaldo, Solange; Ferreira, Joao; Soares, Marcus y Candan, Selami. 2011. New record of Leptoglossus zonatus (Dallas) (Hegeroptera: Coreidae) attacking starfruit (Averrhoa carambola L.) in Sinop, Mato Grosso, Brazil. EntomoBrasilis 4(1): 33-35.

Rodrigues, Daniela y Moreira, Gilson. 2005. Comparative description of the immature stages of two very similar leaf footed bugs, Holymenia clavigera (Herbst) and Anisoscelis foliaceae marginella (Dallas) (Hemiptera, Coreidae, Anisoscelini). Revista Brasileira de Entomología 49(1): 7-14.

Rodríguez, Pedro; Rodríguez, Erick; Romero, Bolívar y Collantes, Rubén. 2011. Relación de la caída de naranjas (Citrus sinensis (L.)) con dos especies de Leptoglossus Guérin-Méneville (Hemiptera: Coreidae) en la Región de Azuero, Panamá. Scientia 21(1): 77-87.

Schaefer, Carl y Mitchell, Paula. 1983. The food plants of the Coreoidea (Hemiptera: Heteroptera). Ann. Entomol. Soc. Amer. 75: 224-233.

Silva, Ricardo; Flores, Patricia y Carvalho, Gervasio. 2001. Descrição dos estágios imaturos de Phthia picta (Drury) (Hemiptera: Coreidae). Neotropical Entomology 30(2): 253-258.

Souza, Carlos y Amaral, Benedicto. 1999a. Nova planta hospedeira de Leptoglossus zonatus (Dallas) (Heteroptera: Coreidae). An. Soc. Entomol. Brasil. 28(4): 753-756.

Souza, Carlos y Amaral, Benedicto. 1999b. Ocorrência Natural de Parasitóides de Leptoglossus ronatus (Dallas) (Heteroptera: Coreidae. An. Soc. Entomol. Brasil. 28(4): 757-759.

Tarango, Socorro; García, Mónica y Candia, María. 2007. Efecto de la alimentación de cinco especies de chinches (Hemiptera: Pentatomidae, Coreidae) en frutos de nogal pecanero. Agric. Téc. Méx. 33(3): 241-249.

Tarango, Socorro. 2013. Manejo integrado de chinches en nogal pecanero. Folleto técnico No. 27. INIFAP, Chihuahua, MX. 35.

Yepes, Francisco; Carmona, Manyer; Correa, Norman y Quiroz, Jhon. 2012. Plagas potenciales del cultivo de Jatropha curcas L., en el Occidente de Antioquía, Colombia. Rev. Fac. Nal. Agr. Medellín 65(2): 6823-6826.

Fecha de recepción: 2 de febrero 2016

Fecha de aceptación: 27 de mayo 2016

\section{Correspondencia}

Rubén Collantes González

rdcg31@hotmail.com 